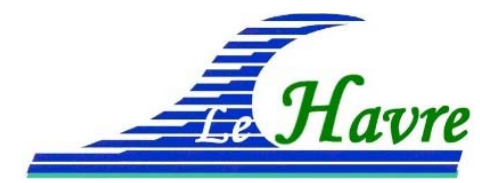

XVI'̀mes Journées Nationales Génie Côtier - Génie Civil

Le Havre, 2020

DOI:10.5150/jngcgc.2020.019 C Editions Paralia CFL

disponible en ligne - http://www.paralia.fr - available online

\title{
Évolution spatio-temporelle du trait de côte du littoral centre Algérien : cas de la baie de Zemmouri
}

\author{
Cherif AOUDJ ${ }^{1}$, Khoudir MEZOUAR ${ }^{1}$, Mokhtar GUERFI ${ }^{2}$, \\ Miloud SALLAYE ${ }^{1}$, Yousra SALEM CHERIF ${ }^{1}$, \\ Abd El Alim DAHMANI ${ }^{1}$, Abdlouhad BOUKHENNAF ${ }^{1}$
}

1. Ecole Nationale Supérieure des Sciences de la Mer et de l'Aménagement du Littoral, ENSSMAL, Bois des Cars, B.P. 19 Dely Ibrahim, 16320, Algérie. cherifaoudj@gmail.com

2. Université des Sciences et des Technologies Houari Boumediene, USTHB, BP 32, El Alia 16111, Bab Ezzouar 16111, Algérie.

\section{Résumé :}

La dynamique du trait de côte est étudiée en Algérie depuis des années en raison des problématiques croissantes liées à la protection des environnements naturels côtiers, mais également de la gestion de l'urbanisme sur le littoral et des risques liés à la mer.

Le littoral de la baie de Zemmouri est le siège d'une urbanisation et d'une activité touristique accrues qui ont conduit à sa fragilisation et au recul de son trait de côte dans la majeure partie de ce secteur depuis 1970. Cette situation s'est aggravée après la construction du port de cap Djinet, la centrale électrique de Zemmouri et les ouvrages de protection de la plage de Boumerdes. Ce travail a pour but de faire progresser la connaissance des milieux côtiers en étudiant l'évolution à long terme des tendances morpho-sédimentaires d'un système côtier anthropisé de la baie de Zemmouri.

L'utilisation des images satellitales multi dates, des photos aériennes et des documents cartographiques existants, acquis avant et après la construction des infrastructures portuaires et urbaines a permis de suivre et de quantifier, en particulier, les transformations qui ont affecté les cotes sableuses, et de mesurer ainsi l'impact des constructions et des ouvrages sur le trait des côtes. Plusieurs outils ont été développés pour calculer la cinématique du trait de côte. Le recours à des méthodes statistiques d'extrapolation ou de calcul de tendance est souvent utilisé en matière d'étude de la dynamique du trait de côte, principalement dans le but d'anticiper l'érosion des côtes. Nous avons donc fait le choix d'en citer un: le Digital Shoreline Analysis System (DSAS). Trois de ces méthodes sont régulièrement utilisées et facilement applicables aux côtes d'accumulation : la méthode des extrémités EPR (End Point Rate), ainsi que la régression linéaire, LRR (Least Regression Rate) et NSM (Net Shoreline Movement). En fonction des différentes échelles temporelles (long, moyen ou court terme), nous avons utilisé ces trois techniques.

Afin de mieux comprendre l'évolution morphologique des cotes sableuses de ce littoral à différentes échelles de temps, sur une longue période, nous avons compilé des positions 


\section{Thème 2 - Dynamique sédimentaire}

de trait de côte à différentes époques et complétées par des relevés sur le terrain ainsi que par des enquêtes auprès des habitants. Cette analyse diachronique du trait de côte, 1972 à 2019, met en évidence un découpage spatial de la plage, en surface d'érosion et d'accrétion, et permet de visualiser l'évolution morphologique dans le temps. Les résultats obtenus montrent une évolution très irrégulière avec des périodes de stabilité, de recul ou d'avancée du rivage.

Mots clés : Baie de Zemmouri, Digital Shoreline Analysis System, Littoral, SIG, Trait de côte.

\section{Introduction}

La problématique de l'érosion côtière occupe une place de plus en plus importante dans la gestion des territoires côtiers à l'échelle de la planète. La compilation de résultats sur l'évolution côtière à l'échelle nationale, continentale et même mondiale démontre un constat similaire : les changements climatiques ont un impact direct sur les milieux côtiers. Au niveau du littoral centre de l'Algérie, en plus d'une augmentation mesurée de l'intensité de l'érosion côtière dans plusieurs secteurs le cadre bâti s'est rapidement étendu sans égard à la sensibilité du littoral. Le littoral s'est ainsi fortement artificialisé au rythme de la mise en place de structures de protection pour lutter contre l'érosion. La baie de Zemmouri qui fait partie de la côte centrale de l'Algérie, est le siège d'une urbanisation et d'une activité touristique accrues qui ont conduit à sa fragilisation et au recul de son trait de côte dans la majeure partie de ce secteur. En effet, elle a connu une importante extension de zones urbaines et industrielles (Cap Djinet, Zemmouri, Boumerdes, Reghaia,...), une intensification de la construction d'unités urbaines et des aménagements portuaires (Zemmouri et Cap Djinet) qui ont largement contribué à sa dégradation. De plus, des rejets d'eaux usées dans l'oued Corso et Isser... et d'eaux chaudes issues de la centrale thermoélectrique installée sur la côte de cap Djinet ont conduit à la dégradation et amplifié le phénomène d'érosion le long des côtes sableuses de la baie. La côte de Zemmouri est continuellement soumise à l'action d'agents naturels d'origine météorologique et marine qui progressivement modifient le paysage littoral. Ces agents morpho dynamiques, principalement les vents, les houles, les courants et la marée, possèdent des caractéristiques spécifiques résultant directement de la situation géographique de la baie et sont directement sous l'influence des conditions atmosphériques et marines provenant de la mer Méditerranée. Comme outil de connaissance, la photo-interprétation est un moyen pour étudier la géomorphologie littorale telle que la genèse des plages, leur pérennité, etc., pour optimiser l'implantation de nouvelles installations et pour suivre et reconstituer l'histoire de ces systèmes côtiers. Une analyse diachronique par photo-interprétation, en utilisant des photographies aériennes des années 1972, 1980, 1993, 1999, 2003 et des images satellitales 2010 et 2017, qui couvrent tout le secteur d'étude, a été réalisée afin d'apprécier la variation spatio-temporelle de la vitesse de l'érosion et d'identifier les principaux facteurs qui sont 


\section{XVİ̀mes Journées Nationales Génie Côtier - Génie Civil \\ Le Havre 2020}

à l'origine du recul, de la stabilité ou, par endroits, de l'avancée du trait de côte des plages sableuses de la baie de Zemmouri.

\section{Méthodologie}

La baie de Zemmouri (ex baie de Courbet) est située au Nord-Est d'Alger, limitée au Nord par la Méditerranée occidentale, au Sud par la plaine de la Mitidja, à l'Est par le Cap Djinet, et à l'Ouest par le Cap Matifou (Figure 1). Elle s'étale sur un linéaire côtier d'environ $54 \mathrm{~km}$, très largement ouverte vers le nord, il est à noter l'existence d'un promontoire rocheux d'environ $100 \mathrm{~m}$ (Rocher Noir).

La zone côtière de Zemmouri est caractérisée par une artificialisation importante liée principalement au développement de la zone industrialo-portuaire de Rouiba, Zemmouri, Cap Djinet. Une partie importante de cette côte reste néanmoins bordée par un cordon dunaire (cordon de Zemmouri), soutenu par de larges estrans composés de sable d'origine marine. L'arrière-pays de cette côte est drainé par de nombreux oueds qui débouchent dans cette baie (Oued Isser, Oued Corso,...).

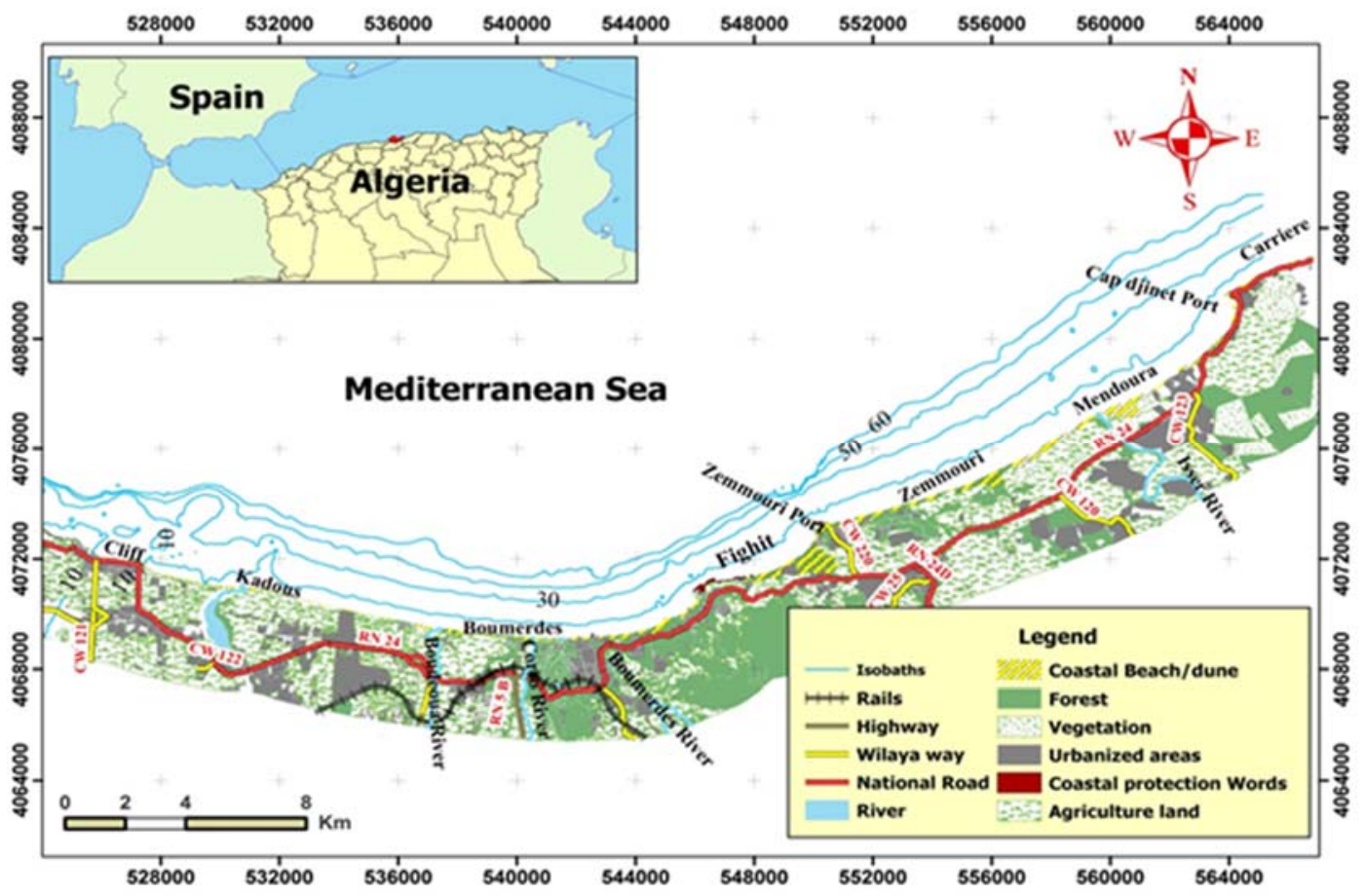

Figure 1. Situation géographique de la Baie de Zemmouri.

\section{Matériels et méthodes}

Afin de mieux comprendre l'évolution morphologique des cotes sableuses de ce littoral à différentes échelles de temps, la méthode adoptée se base sur un ensemble de procédures utilisées par plusieurs auteurs (MENIE OVONO, 2010 ; FAYE, 2010 ; FAYE et al., 


\section{Thème 2 - Dynamique sédimentaire}

2011 ; JUIGNER et al., 2012). Tout d'abord, chaque photographie aérienne a été numérisée à très haute résolution, afin d'obtenir une définition au sol de $50 \mathrm{~cm} /$ pixel. Nous avons compilé des positions de trait de côte à différentes époques et complétées par des relevés sur le terrain ainsi que par des enquêtes auprès des habitants. Cette analyse diachronique du trait de côte, 1972 à 2017, met en évidence un découpage spatial de la plage, en surface d'érosion et d'accrétion, et permet de visualiser l'évolution morphologique dans le temps. Les résultats obtenus montrent une évolution très irrégulière avec des périodes de stabilité, de recul ou d'avancée du rivage.

Dans le cadre de cette étude, on a utilisé des images satellitales ortho-rectifiées, il s'agit de sept images de 1972, 1980, 1993, 1999, 2003, 2010 et 2017. Le traitement des images s'est déroulé en trois étapes :

- D'abord, on a effectué un géo référencement afin de rendre les différentes images superposables.

- Ensuite Le logiciel ArcGIS Desktop 10.3 a été utilisé pour la digitalisation des traits de côte et la réalisation des cartes.

- Enfin DSAS V4.3 a permis le calcul statistique des vitesses d'érosion (EPR), des taux d'évolution (LRR) et les distances entre la ligne de rivage la plus ancienne (1972) et celle qui est la plus récente (2017) à partir des différents traits de côte extraits des images utilisées.

\subsection{Extraction de la ligne de rivage et évaluation d'erreur}

Dans le cadre de cette étude, notre choix s'est porté à définir le trait de côte, par la différence radiométrique entre les pixels sombres relatifs à des parties humides et les pixels claires relatifs à des parties sèches (DOLAN et al., 1980 ; CROWELL et al., 1991; DOUGLAS \& CROWELL, 2000). Il a été numérisé manuellement sur chaque orthophoto-plan des différentes missions à l'aide du logiciel ArcGIS. Avant de procéder à l'analyse des résultats des changements de la ligne de rivage, nous avons d'abord évalué les erreurs relatives à chaque position de la ligne de rivage. En effet, la reprise des photographies aériennes et les mesures automatiques génèrent un certain nombre d'erreurs potentielles associées à la qualité des données utilisées (erreur de pixel (Ep)), géoréférencement des images (erreur quadratique RMSE (ERM)), l'extraction et digitalisation des traits de côte (erreur de numérisation $(E d)$ ) et photo incertitude de la position du niveau de la marée haute (Ev). Ces erreurs sont considérées comme aléatoires et non corrélées, l'erreur totale (Et) est calculée comme la racine carrée de la somme des carrés des différentes variables (équation 1), (FLETCHER et al., 2003).

$\mathrm{Et}(\mathrm{m})=\sqrt{E p^{2}+E R M S^{2}+E d^{2}+E v^{2}}$

L'erreur annualisée a été calculée à partir de l'équation 2 suivante.

$\mathrm{Ea}(\mathrm{m} / \mathrm{an})=\frac{\sqrt{\mathrm{Et}^{2}+\mathrm{Et}^{2}+\mathrm{Et} 3^{2}+\mathrm{Et} 4^{2}}}{\text { Période totale (Nombre } \mathrm{d}^{\prime} \text { années) }}$ 


\section{XVI'mes Journées Nationales Génie Côtier - Génie Civil \\ Le Havre 2020}

\subsection{Calcul des taux d'érosion / accrétion}

Le calcul de l'évolution historique a été réalisé à l'aide du logiciel Digital Shoreline Analysis System, version 4.3, développé par la Commission géologique des États-Unis (USGS). Ce logiciel a permis de générer de manière automatique des transects recoupant les lignes de rivage de 1972 à 2017 tous les $50 \mathrm{~m}$. L'écart entre chacun des tracés est ensuite calculé et rapporté à une variation annuelle, ce qui génère une base de données sur l'évolution historique du trait de côte pour chaque sous période puis pour la période entière. Les changements de positions du littoral sont calculés en utilisant trois techniques d'analyse de données. Le taux des points extrêmes (EPR), le mouvement net de la ligne de rivage (NSM) et le taux net de changement à partir de la régression linéaire (LRR). Les calculs automatiques des taux des changements du trait de côte entre les différentes dates des missions de photographies aériennes (1972- 1993, 1993- 2003, 2003- 2010, 2010-2017 et la période globale entre 1972-2017) ont été opérés à partir des outils de calcul automatisé des taux d'évolution (DSAS).

\section{Résultats}

L'analyse des photographies aériennes de 1972 et 2003 et les images satellitales de 2010 et 2017 a permis de définir la mobilité du trait de côte sur l'ensemble de la côte de Zemmouri. Malgré les erreurs de précision liées à la résolution des photos ainsi que les erreurs de précision introduites dans le traitement de ces photos (ortho rectification et géo référencement), on rappelle qu'une valeur de seuil de mobilité de $5 \mathrm{~m}$ a été considérée pour déterminer les zones en érosion, accrétion ou stables. La mobilité du trait de côte extraite de cette analyse a donc un caractère très affirmé et peut ainsi être prise comme représentative de l'évolution récente durant les deux dernières décennies.

\subsection{Période $1972-1993$}

Les résultats des changements du trait de côte obtenus durant cette période (21 ans) sur l'ensemble des transects de la baie de Zemmouri montrent une position de recul avancée du trait de côte tout au long de la baie. Les forts taux de recul en terme d'EPR enregistrés durant cette période varient entre $-0.6 \mathrm{~m} /$ an et $-1.2 \mathrm{~m} / \mathrm{an}$ au niveau de Ain Taya (les Flots Bleus, Surcouf, Deca-plage, Tarfaia, El Kadous) et des valeurs entre $-0.5 \mathrm{~m} / \mathrm{an}$ et au niveau de Boudouaou El Bahri et Corso, ces formes d'érosion sont causées par les agents hydrodynamiques, concentration de l'énergie de la houle dans cette zone qui donne naissance à des courants de retour et de dérive littorale qui déclenchent un transit littoral vers l'Ouest. Au niveau de la partie Est (plage de Zemmouri jusqu'à la plage de Cap Djinet), on distingue des formes d'accrétion avec des vitesses de $1 \mathrm{~m} / \mathrm{an}$ à $3 \mathrm{~m} / \mathrm{an}$. Cela est dû généralement à la dissipation des énergies de la houle au voisinage de la côte qui favorise l'accumulation des sables et la formation des plages. 


\section{Thème 2 - Dynamique sédimentaire}

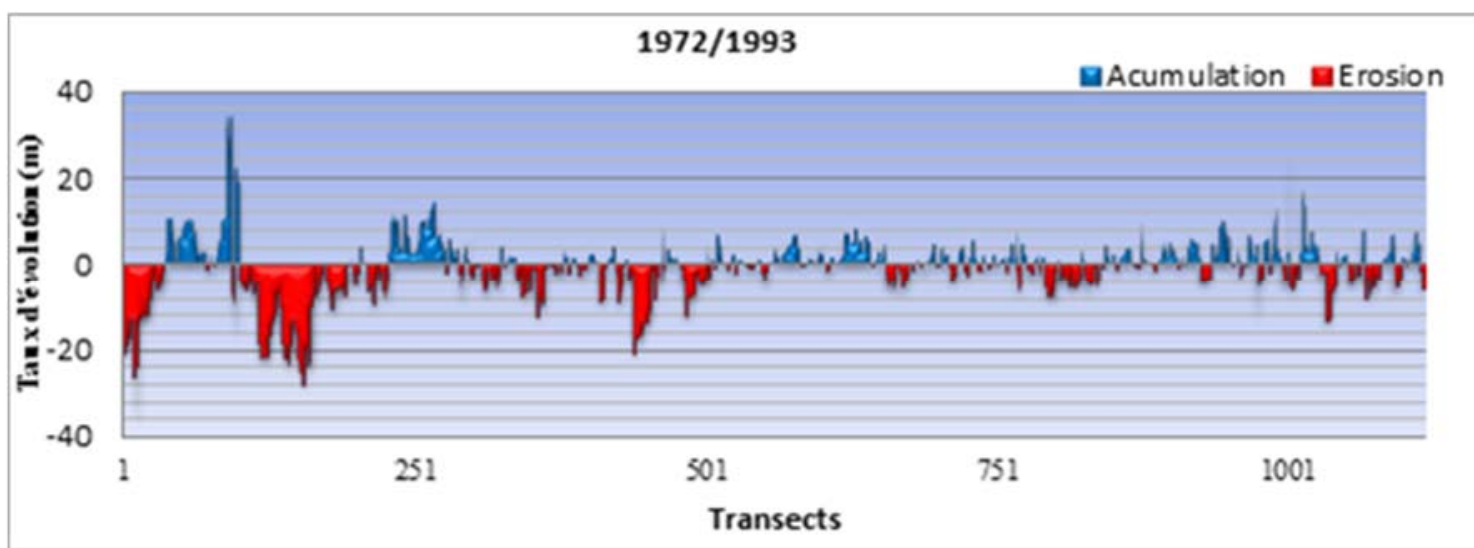

Figure 2. Evolution moyenne du rivage dans la Baie de Zemmouri durant 1972-1993.

\subsection{Période 1993- 2003}

Durant cette courte période de temps (10 ans) la zone occidentale de la baie de Zemmouri (Cap Matifou - Oued Reghaia) est marquée par une phase d'érosion généralisée, à l'exception des plages de Zerzouria et Kef Ain-Taya qui ont enregistré une progradation. La valeur moyenne globale d'EPR calculée a atteint le chiffre de $-0.46 \mathrm{~m} / \mathrm{an}$. Cette période de 6 ans allant de 1993 à 1999 est caractérisée par accentuation du phénomène d'érosion comparativement à la période précédente de 1980 à 1993. La majorité des plages sont touchées par le recul, Ain Beida, Surcouf, Déca-plage, Boumerdes, Zemmouri, Djinet avec des taux maximum de recul d'EPR de $-2.99 \mathrm{~m} / \mathrm{an},-1.74 \mathrm{~m} / \mathrm{an},-3.66 \mathrm{~m} / \mathrm{an}$ et $-1.98 \mathrm{~m} /$ an respectivement. L'engraissement par les sédiments a continué au niveau des plages de Tamaris et El Kadous avec un taux de progradation moyen (EPR) de 1.13 m/an. L'accrétion maximale est enregistrée au niveau de Tamaris et El Kadous avec des valeurs maximales d'EPR de 2,72 et $2.73 \mathrm{~m} / \mathrm{an}$ respectivement.

\subsection{Période 2003-2010}

Durant cette période l'évolution de la ligne du rivage de la baie de Zemmouri affiche une position recul-avancée tout au long de toute la côte. Au niveau de la partie Ouest de la plage Zerzouria, le rivage marque un important recul du trait de côte avec des taux d'EPR qui varient entre $-0.8 \mathrm{~m} /$ an et $-2 \mathrm{~m} / \mathrm{an}$. Cependant sa partie orientale transects a enregistré une avancée du trait de côte avec des taux d'EPR de $0.72 \mathrm{~m} / \mathrm{an}$ à $2 \mathrm{~m} / \mathrm{an}$. Dans la partie occidentale plage Surcouf, Déca-plage et El Kadous le trait de côte montre une situation de recul-avancée-recul. Le recul le plus marqué de la côte est enregistré au niveau de la plage Surcouf et au niveau de la plage El Kadous qui a marqué un recul généralisé sur toute son étendue (Figure 4). Les valeurs d'EPR enregistrées varient entre $-0.5 \mathrm{~m} / \mathrm{an}$ et $2.0 \mathrm{~m} / \mathrm{an}$. 


\section{XVİ̀mes Journées Nationales Génie Côtier - Génie Civil \\ Le Havre 2020}

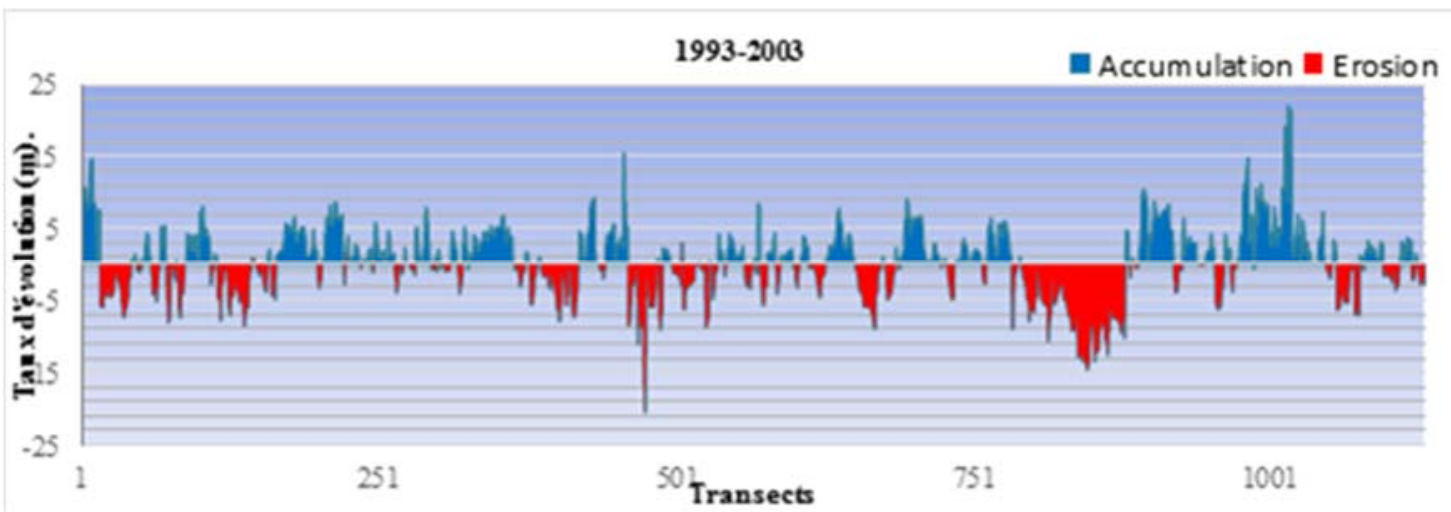

Figure 3. Evolution moyenne du rivage dans la Baie de Zemmouri durant 1993-2003.

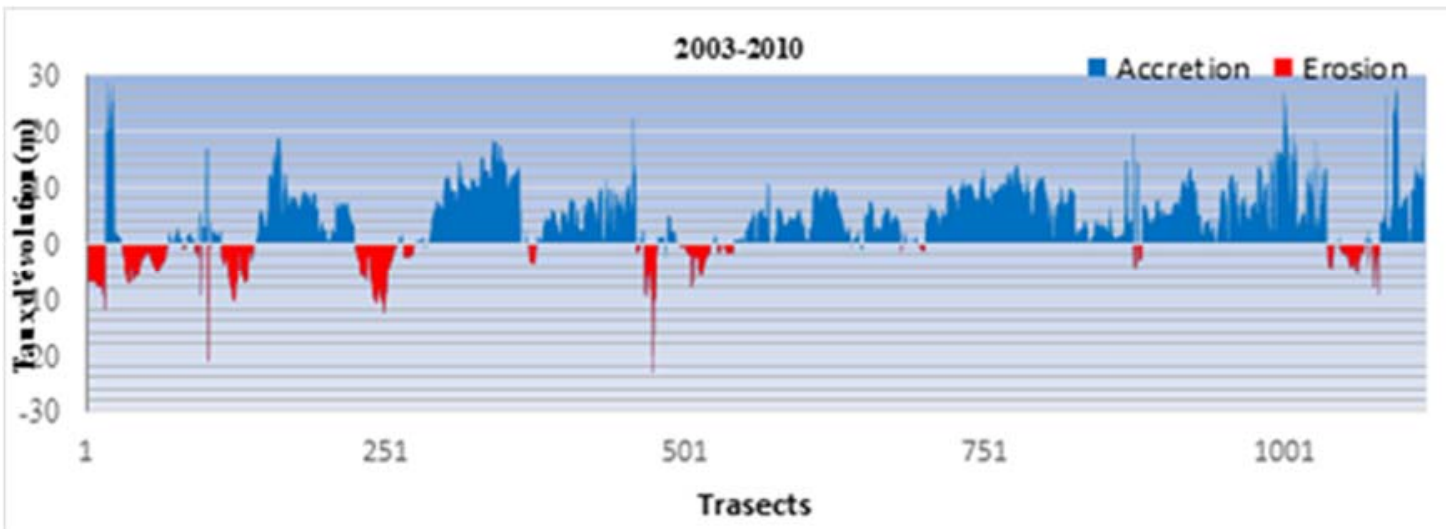

Figure 4. Evolution moyenne du rivage dans la Baie de Zemmouri durant 2003-2010.

\subsection{Période 2010-2017}

Cette période allant de 2007 à 2017 est caractérisée par une forte augmentation du phénomène d'érosion par rapport aux périodes précédentes (1972-1993, 1993-2003 et 2003-2010). Les résultats de l'analyse statistique des changements du trait de côte affichent un recul moyen d'EPR de $-0.2 \mathrm{~m}$ /an sur la totalité de la ligne du rivage. Dans la partie est de cette portion côtière les résultats obtenus affichent des zones en accrétion avec des taux de progradation de $0.2 \mathrm{~m} /$ an (Figure 5). L'engraissement par les sédiments a continué au niveau de la plage de d'El Kadous avec un taux de progradation moyen (EPR) de $0.9 \mathrm{~m}$ /an. L'érosion moyenne maximale (érosion forte à très forte) est enregistrée sur les plages de Boudouaou el Bahri jusque la plage de Zemmouri Est, avec des valeurs maximales d'EPR de $-1.64 \mathrm{~m} / \mathrm{an}$ et $-3.59 \mathrm{~m} /$ an respectivement. Les causes de ce recul peuvent être dues sont à la fois à des facteurs hydrodynamiques (houle et courants), comme le montre la modélisation de la houle sur la zone de Zemmouri, ou on a constaté des fortes concentrations des énergies de la houle au voisinage de la côte qui engendre des courants de dérive littorale dans ces zones. 


\section{Thème 2 - Dynamique sédimentaire}

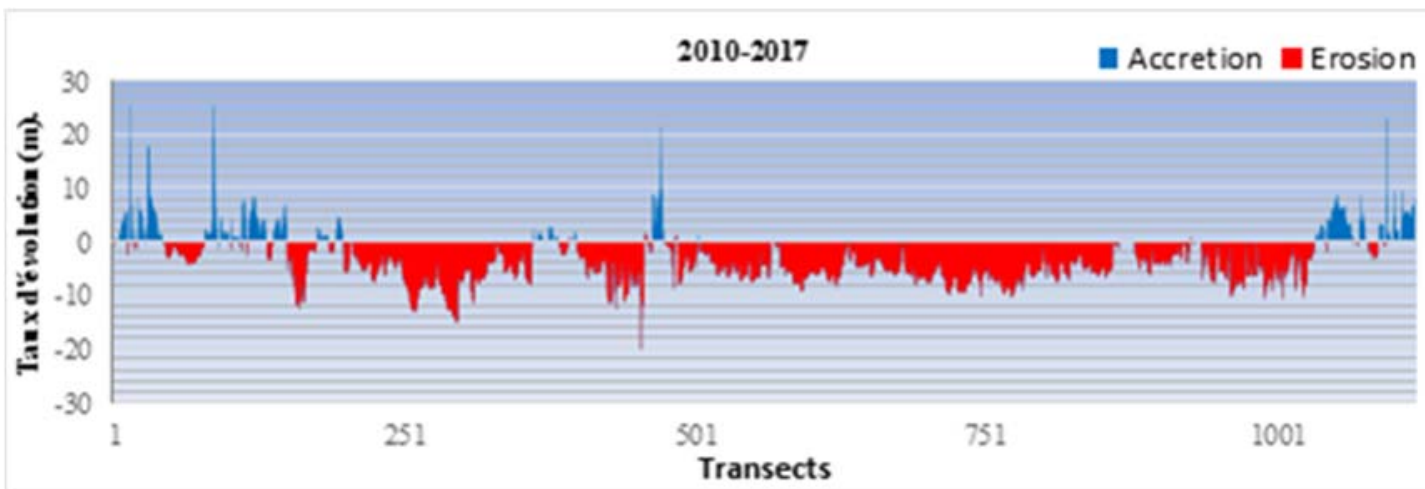

Figure 5. Evolution moyenne du rivage dans la Baie de Zemmouri durant 2010-2017.

\section{Conclusions}

Les résultats d'analyse des changements de la position de la ligne du rivage au cours de ces 45 dernières années (1972-2017) ont révélé que la côte de la baie de Zemmouri est soumise soit à l'érosion ou à l'accumulation en fonction des secteurs. Les taux net moyens de recul calculé à partir de la méthode EPR, varie entre $-0.3 \mathrm{~m} / \mathrm{an}$ et $-2 \mathrm{~m} / \mathrm{an}$. Au niveau de la partie Est de la baie, on constate des accumulations au voisinage du port de Cap Djinet, généralement due au flux sédimentaire engendré par les courants de dérive littorale Est et Ouest, les sédiments sont piégés entres les ouvrages de protection et la jetée du port. Les plages les plus touchées par l'érosion sont celles de la partie occidentale (plage El Kadous jusqu'à la plage de Boumerdes) avec des taux de recul d'EPR qui varient de -0.9 à $-1,8 \mathrm{~m} /$ an soit $-40 \mathrm{~m}$ à $-100 \mathrm{~m}$. Les plages de la partie centrale (plage de Zemmouri Est jusqu'à la plage de Dar El Mendil) à leur tour enregistrent un recul important, soit une érosion de l'ordre de- $0,5 \mathrm{~m} / \mathrm{an}$ à $-2 \mathrm{~m} / \mathrm{an}$. Les plages de Boumerdes, Sahel Brouk, Figuier Est, Figuier Ouest, Sghiret et Sable d'or connaissent également un recul entre ces deux dates, mais de moindre ampleur, l'érosion n'atteignant qu'environ $45 \mathrm{~m}$ soit un taux de recul qui varie entre -0.5 à $-1 \mathrm{~m} / \mathrm{an}$.

Les causes de ce recul peuvent être dues à la fois à des facteurs naturels et anthropiques. Les causes naturelles d'érosion dans les plages de la baie de Zemmouri sont dues principalement aux effets répétés et cumulés des tempêtes et dans la hausse relative du niveau moyen de la mer. Des enregistrements marégraphiques indiquent un relèvement au niveau de la mer Méditerranée de l'ordre de 1.2 à $1.5 \mathrm{~mm}$ par an depuis environ un siècle. Les causes anthropiques peuvent être dues aux extractions massives et arbitraires des quantités requises pour l'essor urbain que connaît la Wilaya d'Alger depuis le début des années 1970. Pour répondre à cette forte demande plusieurs sites d'extraction de sables ont été ouverts le long de la côte de la Wilaya de Boumerdes. Ces extractions ont touché simultanément les lits d'oueds, les plages et le cordon dunaire de Zemmouri qui a été complètement détruit. D'après des sorties sur terrain, nous avons constaté clairement que les prélèvements de sable effectués directement sur les plages et les cordons dunaires ont eu sans aucun doute des impacts négatifs qui ont lourdement affecté l'environnement 


\section{XVIèmes Journées Nationales Génie Côtier - Génie Civil \\ Le Havre 2020}

côtier des plages de la côte. Il faut noter également la réduction des débits solides des Oueds (Corso, Isser, Boumerdes,...) durant les dernières décennies vu l'extraction excessive des sables sur les lits de ces oueds.

\section{Références bibliographiques}

CROWELL M., LEATHERMAN S.P., BUCKLEY M.K. (1991). Historical shoreline change: Error analysis and mapping accuracy, Journal of Coastal Research, Vol. 7(3), pp 839-852. https://www.jstor.org/stable/4297899

DOLAN R., HAYDEN B., MAY P., MAY S. (1980). The reliability of shoreline change measurements from aerial photographs, Shore and Beach, Vol. 48(4), pp 22-29.

DOUGLAS B.C., CROWELL M., (2000). Long-term shoreline position prediction and error propagation. Journal of Coastal Research, Vol. 16(1), pp 145-152. https://www.jstor.org/stable/4300019

FAYE I., GIRAUDET E., GOURMELON F., HENAFF A. (2011). Cartographie normalisée de l'évolution du trait de côte. Mappemonde 104, 12 p. http://mappemonde.mgm.fr/num32/articles/art11404.html

FAYE I. (2010). Dynamique du trait de côte sur les littoraux sableux de la Mauritanie à la Guinée-Bissau (Afrique de l'Ouest) : Approches régionale et locale par photointerprétation, traitement d'images et analyse de cartes anciennes. Thèse de doctorat, Université de Bretagne Occidentale, 321 p.

FLETCHER C.H., ROONEY J., BARBEE M., LIM S.C., RICHMOND B. (2003). Mapping shoreline change using digital orthophotogrammetry on Maui, Hawaii. Journal of Coastal Research, Special Issue n³8, pp 106-124. https://www.jstor.org/stable/25736602 JUIGNER M., ROBIN M., FATTAL P., MAANAN M., LE GUERN C., GOUGUET L., BAUDOUIN V., DEBAINE F. (2014). Cinématique d'un trait de côte sableux en Vendée entre 1920 et 2010.In "L'homme et la dynamique littorale : maîtrise ou adaptation", sous la coordination de Y. Lageat, Y. Battiau-Queney et M.-C.Prat, LGPA Editions, Dynamiques Environnementales, n³0, pp 29-39.

MENIE OVONO Z., (2010). Évolution de la flèche Mandji de l'holocène a l'actuel. Analyse et cartographie du risque côtier, Université de Nantes, Thèse de Doctorat, 301 p. 
Thème 2 - Dynamique sédimentaire 